\title{
Range and Battery Depletion Concerns with Electric Vehicles
}

\author{
Tomio Miwa, ${ }^{1}$ Hitomi Sato, ${ }^{2}$ and Takayuki Morikawa ${ }^{2}$ \\ ${ }^{1}$ Institute of Materials and Systems for Sustainability, Nagoya University, Furo-cho, Chikusa-ku, Nagoya 464-8603, Japan \\ ${ }^{2}$ Institute of Innovation for Future Society, Nagoya University, Furo-cho, Chikusa-ku, Nagoya 464-8603, Japan \\ Correspondence should be addressed to Tomio Miwa; miwa@nagoya-u.jp
}

Received 22 March 2017; Revised 15 September 2017; Accepted 3 October 2017; Published 31 October 2017

Academic Editor: Stephen Boyles

Copyright (C) 2017 Tomio Miwa et al. This is an open access article distributed under the Creative Commons Attribution License, which permits unrestricted use, distribution, and reproduction in any medium, provided the original work is properly cited.

\begin{abstract}
This study investigates the effects of the range of a battery electric vehicle (EV) by using questionnaire data. The concern about battery depletion changes according to charging station deployment. Firstly, the methodology for deriving the probabilistic distribution of the daily travel distance is developed, which enables us to analyze people's tolerance of the risk of battery depletion. Secondly, the desired range of an EV is modeled. This model considers the effect of changing charging station deployment and can analyze the variation in the desired range. Then, the intention of a household to purchase an EV is analyzed by incorporating range-related variables. The results show that people can live with a risk of battery depletion of around $2 \%$ to $5 \%$. The deployment of charging stations at large retail facilities and/or workplace parking spaces reduces the desired range of an EV. Finally, the answers to the questionnaire show that the probability of battery depletion on a driving day has little effect on the intention to purchase an EV. Instead, people tend to evaluate the range by itself or directly compare it with their desired range.
\end{abstract}

\section{Introduction}

With increasing awareness of environmental issues, governments are putting in place policies to promote battery electric vehicles (EVs). The Japanese government has set the goal of increasing the ratio of new-generation vehicles in domestic sales of new cars to $20 \%$ by 2020 . According to this policy, the ratio of EVs is expected to reach $15 \%$ by that year. However, EVs will account for only $0.4 \%$ of sales in 2017 [1]. Hence, although governments are implementing subsidy programs to promote EVs, research shows that more subsidies are necessary for significant promotion [2-7].

The literature points out that one of the major reasons for EVs not becoming more widely popular is their limited range [8-11]. Adepetu and Keshav [12] found that while increasing the capacity of EV batteries would reduce range anxiety and make EVs more attractive for purchase, the current costs are still prohibitive. Thanks to recent advances in battery technology, range is improving rapidly and can now exceed $250 \mathrm{~km}$ according to some specifications [13]. However, actual driving range is generally much shorter because of the use of air conditioning, number of passengers, and need to retain a margin before the next charge [14]. This means that the range of an EV is much less than that of a gasoline car, which is typically around $500 \mathrm{~km}$. To gain greater market penetration, there is thus a need to relieve drivers' concerns about range.

To obtain knowledge about how such concerns can be reduced, the authors analyzed driver concern about vehicle range by using the questionnaire survey data. In particular, we analyzed the influence of the short range of an EV on the preference for EVs. For this purpose, we derived the probabilistic distribution of the daily travel distance and analyzed people's tolerance of the risk of battery depletion. After that, the desired range of an EV was modeled and the effect of the deployment of charging stations examined. Then, the intention of households to purchase an EV was analyzed by incorporating range-related variables.

The remainder of this paper is organized as follows. In Section 2, the literature related to this subject is reviewed. Section 3 outlines the questionnaire data and presents the summary statistics of the data obtained. Section 4 describes the methodologies employed in the analysis, which include the daily travel distance distribution model and desired EV range model. The results of the analysis are then discussed. Section 5 applies an EV purchasing model incorporating range-related variables and discusses the findings. Finally, 
Section 6 presents the conclusions and discusses future research directions.

\section{Literature Review}

Many studies have investigated the impact of the short range and other characteristics of EVs on purchasing and use intentions. In this section, the authors briefly summarize this existing research and clarify the position of this study related to the literature. In this review, the literature is classified into driving data analysis and questionnaire/interview data analysis.

2.1. Driving Data Analysis. Greene [15] analyzed potential demand for EVs from the aspect of the daily travel distance. The distribution of the daily travel distance was modeled based on a gamma distribution and distribution parameters were estimated. This study found significant potential demand for EVs even if their range is relatively short $(160 \mathrm{~km})$. On the contrary, Pearre et al. [16] analyzed the distance traveled by 484 cars in one year and showed that $91 \%$ of the sample had the possibility to exceed $160 \mathrm{~km}$ in range. Woodjack et al. [17] analyzed EV users' perceptions by using data from an EV trial use experiment with 235 households in Los Angeles and New York. They focused on the short range and long charging times as typical shortcomings of EVs and reported that $81 \%$ of the participants had cancelled a trip because of their vehicle's short range.

Franke and Krems [9] investigated the changes in the daily travel distance and desired range before and after a field experiment with 79 households. They reported that the desired range is much greater than the usual distance traveled in a day. In addition, the desired range tends to fall after the long-term use of an EV. Similarly, Rauh et al. [18] used the same data and reported that the actual use of an EV can reduce the desired range. Tal et al. [19] analyzed the travel data of 3,500 plug-in hybrid EVs and showed that a vehicle with a larger battery tends to be charged away from home and used for longer distances. Greaves et al. [14] analyzed the driving data for 166 vehicles and concluded that an EV with a range as low as $60 \mathrm{~km}$ and a charging station at home can accommodate well over $90 \%$ of day-to-day driving. Stark et al. [20] analyzed similar GPS data and found that $58 \%$ of cars can be replaced even if no fast charging is available. Sun et al. [21] analyzed the driving data from an EV usage trial conducted in Japan and showed that range anxiety arises mainly from the low number of charging stations and unfamiliarity with the charging infrastructure. Li et al. [22] developed a mixture distribution model to describe the probabilistic distribution of daily travel distance of taxi drivers in Beijing. Based on the massive trip records of taxi, they analyzed the EV range which satisfied the daily travel distance of taxis and show the usefulness of understanding the travel distance distribution.

From the above review, it is found that while the short range of EVs can cover a considerable amount of daily travel, it cannot cover all daily travel. Although data on the actual daily travel distance are useful for understanding potential demand for EVs and for reaching other meaningful findings, the accessibility of such data is limited in Japan.
Therefore, it is valuable to develop the methodology for deriving the probabilistic distribution of daily travel distance based on questionnaire survey data and to incorporate it in the analysis.

2.2. Questionnaire/Interview Data Analysis. Bunch et al. [23] analyzed intention to purchase a next-generation vehicle using stated preference (SP) data and showed that a short range significantly reduces the price that people are willing to pay. On the contrary, Kurani et al. [24] analyzed data from interactive interviews based on travel diaries. They concluded that consumers' desired range of EVs is substantially lower than those shown in past research and found evidence of a viable market for an EV with a driving range of only 60 to 100 miles. Chéron and Zins [25] conducted a conjoint analysis to evaluate EV characteristics and showed that long charging times present an obstacle to wide market dissemination.

Golob and Gould [26] conducted a questionnaire survey before and after a long-term EV use experiment and showed that the desired range is more than double the usual average range. Plötz et al. [27] investigated early adopters of EVs in Germany by analyzing questionnaire data and concluded that early adopters live in suburbs or rural areas and use EVs for commuting. This finding indicates that early adopters expect an economic benefit because of their long mileage. Jensen et al. [28] conducted a stated choice experiment before and after EV usage. They showed that the willingness to use an EV declines after experience, particularly if the range of the $\mathrm{EV}$ is short. A similar finding was reported by Skippon et al. [29].

Kim et al. [30] conducted a web-based survey for the participants of an EV sharing program and pointed out that participants' social and economic perspectives are the most important factors affecting attitudes toward EV purchasing behavior. Junquera et al. [31] conducted a logistic regression analysis by using the questionnaire survey data obtained from 1,245 Spanish respondents to analyze the relationship between consumers' profile and their intentions to purchase an EV. They found that perceptions of price, battery lifetime, charging time, and range all explain willingness to purchase an EV. Beck et al. [32] conducted an online questionnaire that asked respondents to state their most and least preferred cars and showed that their preference for the range of an EV is significantly influenced by their environmental concerns.

Axsen et al. [33] compared the preferences for an EV of early adopters and potential buyers and found that early adopters are more likely to be multivehicle households that can adapt to the limited range of an EV. Franke et al. [34] analyzed data from EV users and reported that daily mobility patterns, the percentage of travel not coverable because of the limited EV range, and the actual usable range drive range satisfaction. Javid and Nejat [35] conducted a multiple logistic regression analysis by using household travel data in California. Their research showed that a household's income and charging station density have significant impacts on EV adoption.

The literature shows mixed findings. Therefore, the effect of an EV's range remains open for discussion. In addition, no research has investigated the effect of the probability of 
battery depletion on the intention to purchase an EV. In this study, the authors develop a methodology for deriving the probabilistic distribution of the daily travel distance and model the desired range of an EV. Then, the intention to purchase an EV is analyzed based on this information.

\section{Data}

3.1. SP Questionnaire Survey. The data used in this study were collected by a questionnaire survey carried out in November 2011 around Nagoya City, Japan. A total of 4,200 questionnaires were distributed randomly in mailboxes and 880 returns were obtained. The number of questionnaires with the key questions completed was 394. The purpose of this survey was to predict mass demand for EVs in the Nagoya metropolitan area [36, 37]. The methodologies and viewpoints applied in the past researches of the authors were different from this study.

Table 1 outlines the survey questions and provides the summary statistics. The questionnaire consisted of four parts. The first part included questions on car ownership and use. The second explained the advantages and disadvantages of EVs. The advantages explained in the questionnaire included the economical mileage cost and zero emissions while driving. The disadvantages included short vehicle range and long charging time. All advantages and disadvantages were shown with actual values. In addition, values for gasoline cars were shown as well for comparison. Then, we asked about the preferred deployment of charging stations and desired EV range (nine range categories: up to $50 \mathrm{~km}, 50-100 \mathrm{~km}$, $\ldots, 350-400 \mathrm{~km}$, and over $400 \mathrm{~km}$ ) with and without the preferred deployment. In the third part, the stated preferences in terms of purchase were surveyed based on EVs with hypothetical features. Lastly, the questions in the fourth part related to house and household characteristics.

The first part about car use collected three pieces of key information: the most frequent daily travel distance (mode value of the daily travel distance) on a driving day, how often the car was used, and how often a trip was more than twice as long as the most frequent distance. The purpose of collecting these three pieces of information was to obtain the distribution parameters of the daily travel distance for each owned car. This analysis has not been conducted in past research. In addition, since the daily travel distance is always a positive value and its distribution is skewed, it is thought that its average and variance values cannot be answered intuitively. That is, asking the most frequent daily travel distance is convenient both to obtain reliable answers and to derive the probabilistic distribution of the daily travel distance. The derivation of the distribution parameters from the answers is discussed in Section 4.2.

The hypothetical EV features (factors) used in the third part were set by using an orthogonal table with seven factors at three levels. The factors considered included capacity, price, charging time, range, the deployment ratio of charging stations at gas stations, rest areas on intercity expressways, and large retail facilities. The intention to purchase an EV with the hypothetical features was asked twice for different hypothetical features sets. In the hypothetical features sets,

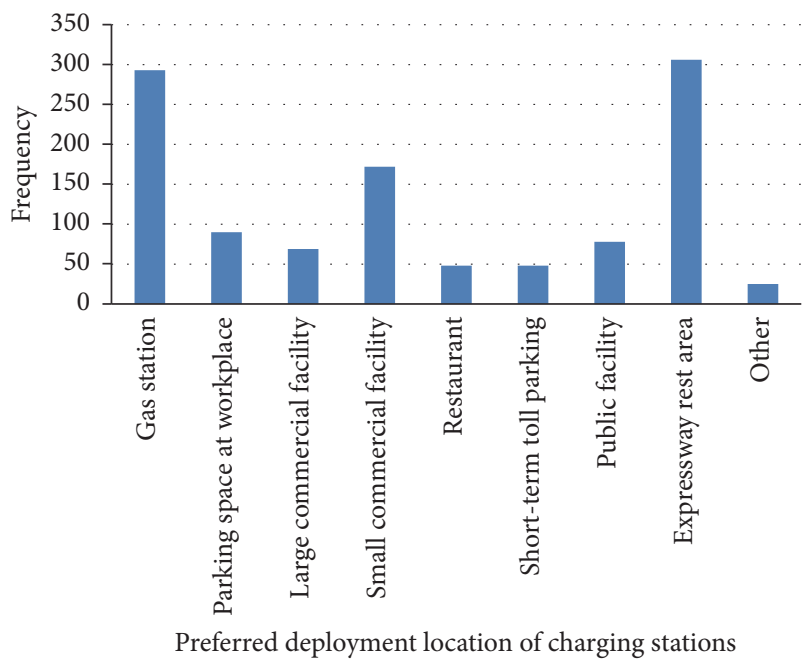

Figure 1: Preferred deployment of charging stations (maximum three answers).

only dependency of price and capacity was considered. However, the dependency between other features exists in reality (e.g., the price of an EV with a longer range is higher; $[12])$. This is one of the limitations of this study.

3.2. Summary Statistics. The summary statistics in Table 1 show that 1.48 cars are owned per household in the survey area and $89 \%$ of those cars have a gasoline engine. The average value of the most frequent daily travel distance per driving day is $21.5 \mathrm{~km}$ on weekdays and $35.7 \mathrm{~km}$ on weekends and holidays. More than twice the most frequent travel distance is traveled on one or two weekdays and one or two weekend days/holidays per month. Since in the questionnaire it was assumed that the number of weekdays in a month is 20 and that of weekend days/holidays is 8 , those mean $5-10 \%$ and $10-20 \%$, respectively.

Figure 1 shows the answers for preferred charging station locations. Deployment at gas stations and expressway rest areas stands out. This finding means that people feel the need for charging stations in easily accessible locations that are convenient in daily life and when on a long drive. Among other preferred locations, there is a desire for charging stations at small retail facilities such as supermarkets. Figure 2 shows the desired range of an EV with and without the deployment of charging stations at the preferred locations. The case without the deployment of charging stations means the actual deployment situation at the time of the survey. As shown, there is little or no deployment for all location types. The most desired vehicle range is $200-250 \mathrm{~km}$ without the deployment of charging stations, but this falls to $100-150 \mathrm{~km}$ with the preferred deployment. The difference between these two distributions is statistically significant $\left(\chi^{2}=84.7 ; \mathrm{df}=\right.$ $\left.8 ; \chi_{8}^{2}(0.01)=20.1\right)$. This test result means that the preference for the range of an EV changes according to the circumstances surrounding charging station deployment.

Returning to Table 1, the intention to purchase an EV with the hypothetical features is around $40 \%$ for the two questions. 


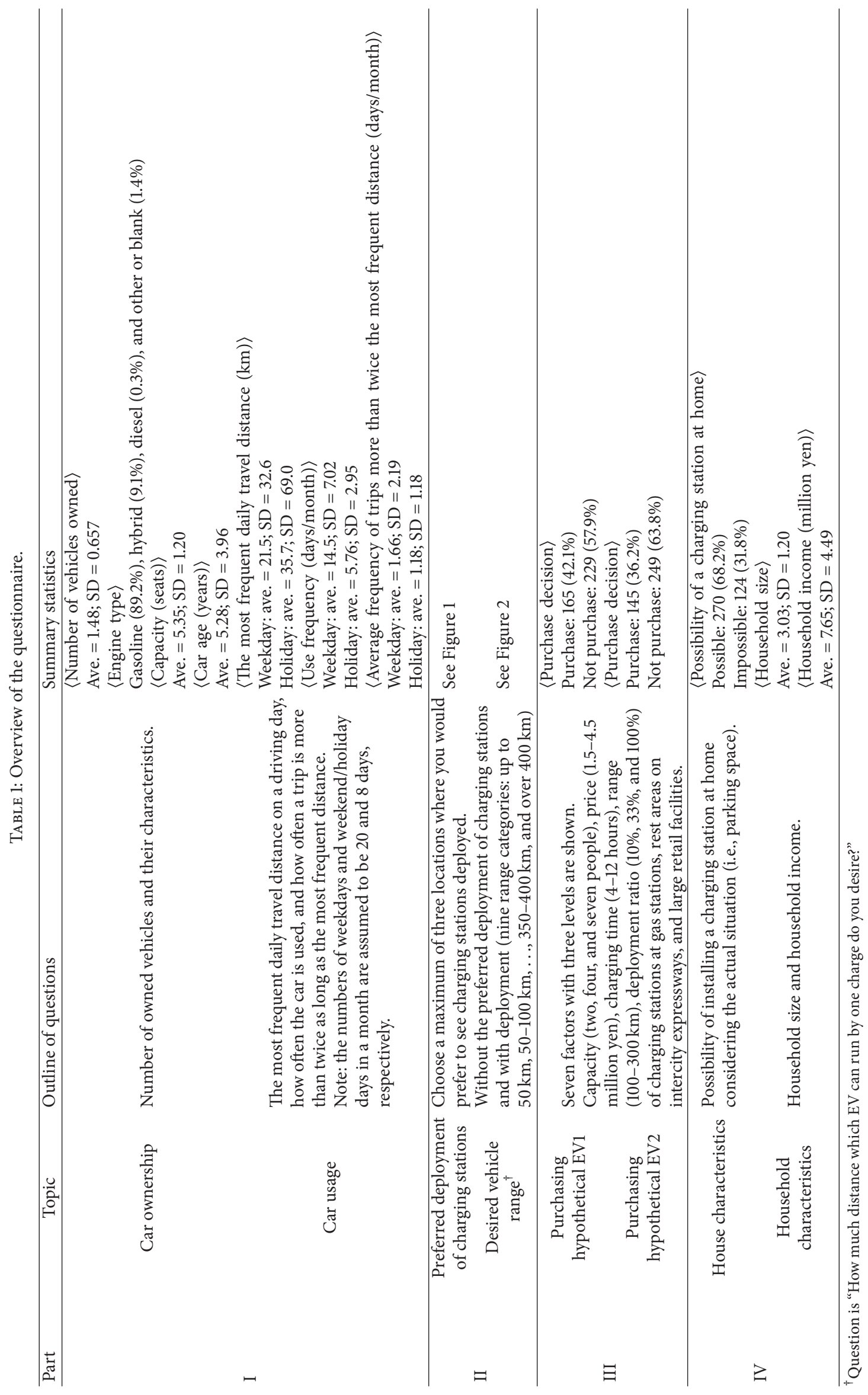




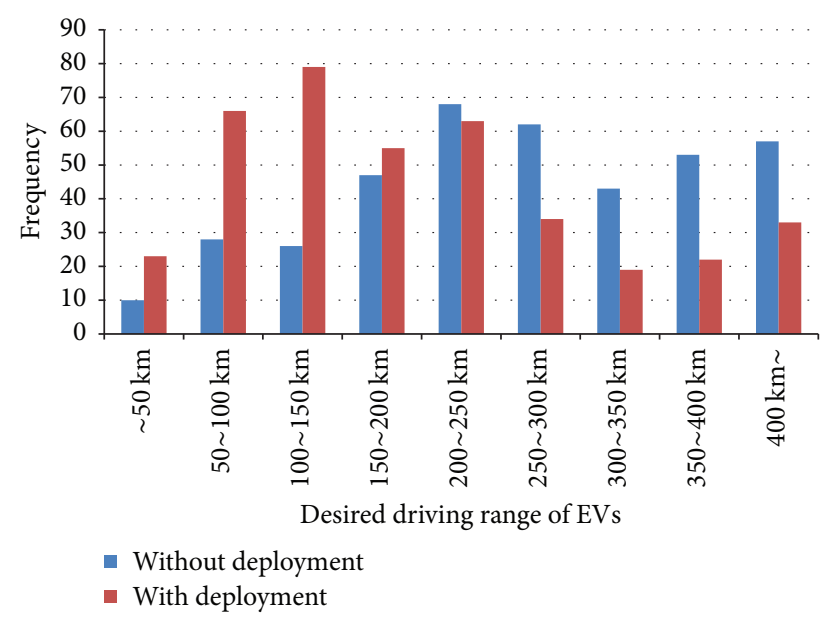

FIGURE 2: Desired range with and without the preferred deployment of charging stations.

In addition, $68.5 \%$ of participants consider it possible to have a charging station at home.

\section{Methodology and Analysis}

4.1. Household's Daily Travel Distance Distribution. The travel distance must have a positive value. As its statistical distribution, gamma, Weibull, and log-normal distributions have been applied in the literature $[38,39]$. In this study, Weibull and log-normal distributions are applied in the analysis because of their usability. Assuming that the daily travel distance follows a Weibull or log-normal distribution, the parameters of the distribution can be obtained from each household's most frequent daily travel distance, car use frequency, and the frequency of traveling more than twice the most frequent distance.

4.1.1. Weibull Distribution for the Daily Travel Distance and Battery Depletion Probability. In the case that the daily travel distance is assumed to follow a Weibull distribution, its distribution parameters can be derived from the questionnaire data as follows. For car $m$ in household $n$ on a driving day of type $w$ (weekday or weekend/holiday), the probabilistic density function of the daily travel distance $x_{w, m, n}$, which follows a Weibull distribution, can be expressed as follows:

$$
\begin{aligned}
f_{w} & \left(x_{w, m, n}\right) \\
= & \frac{k_{w, m, n}}{\lambda_{w, m, n}}\left(\frac{x_{w, m, n}}{\lambda_{w, m, n}}\right)^{k_{w, m, n}-1} \exp \left\{-\left(\frac{x_{w, m, n}}{\lambda_{w, m, n}}\right)^{k_{w, m, n}}\right\},
\end{aligned}
$$

where $f_{w}(\cdot)$ is the probability density function of the Weibull distribution, while $\lambda_{w, m, n}$ and $k_{w, m, n}$ are the scale parameter and shape parameter, respectively.

The distribution form depends on the value of the shape parameter $k_{w, m, n}$. In the case that it is larger than one, the mode value of the distribution becomes larger than zero. In other cases, the mode value is zero. Since the value of $x_{w, m, n}$ is the daily travel distance on a driving day in this study and its mode value (i.e., the most frequent daily travel distance) is larger than zero, the value of $k_{w, m, n}$ must be larger than one. In this case, the following equation regarding the mode value of the Weibull distribution can be obtained:

$$
x_{w, m, n}^{*}=\lambda_{w, m, n}\left(\frac{k_{w, m, n}-1}{k_{w, m, n}}\right)^{1 / k_{w, m, n}},
$$

where $x_{w, m, n}^{*}$ is the mode value of the Weibull distribution and this is the most frequent daily travel distance on a driving day in this study.

Let $y_{w, m, n}$ be car use frequency and let $y_{w, m, n}^{\prime}$ be the frequency of traveling more than twice the most frequent daily travel distance. Then, the probability of traveling more than twice the most frequent daily travel distance can be expressed as follows:

$$
\frac{y_{w, m, n}^{\prime}}{y_{w, m, n}}=\exp \left\{-\left(\frac{2 x_{w, m, n}^{*}}{\lambda_{w, m, n}}\right)^{k_{w, m, n}}\right\}
$$

By substituting (2) into (3), the following equation using only the shape parameter $k_{w, m, n}$ is obtained:

$$
\ln \left(\frac{y_{w, m, n}^{\prime}}{y_{w, m, n}}\right)=-2^{k_{w, m, n}}\left(\frac{k_{w, m, n}-1}{k_{w, m, n}}\right)
$$

The value of $k_{w, m, n}$ which satisfies (4) can be obtained by solving it numerically. The value of the scale parameter $\lambda_{w, m, n}$ can be obtained by substituting the obtained value of $k_{w, m, n}$ into (2).

It is assumed that an EV is fully charged at home in the morning and then is not recharged until the end of the day. In this case, the probability that an EV with range $R$ experiences battery depletion, $P_{\text {Depletion }}^{W}\left(R \mid \lambda_{w, m, n}, k_{w, m, n}\right)$, is calculated as follows:

$$
\begin{aligned}
& P_{\text {Depletion }}^{W}\left(R \mid \lambda_{w, m, n}, k_{w, m, n}\right) \\
& \quad=\exp \left\{-\left(\frac{R}{\lambda_{w, m, n}}\right)^{k_{w, m, n}}\right\} .
\end{aligned}
$$

4.1.2. Log-Normal Distribution for the Daily Travel Distance and Battery Depletion Probability. In the case that the daily travel distance is assumed to follow a log-normal distribution, its distribution parameters can be derived from the questionnaire data as follows. For car $m$ in household $n$ on a driving day of type $w$ (weekday or weekend/holiday), the probabilistic density function of the daily travel distance $x_{w, m, n}$, which follows the log-normal distribution, can be expressed as follows:

$$
\begin{aligned}
& f_{L N}\left(x_{w, m, n}\right) \\
& =\frac{1}{\sqrt{2 \pi} \sigma_{w, m, n} x_{w, m, n}} \exp \left\{-\frac{\left(\ln x_{w, m, n}-\mu_{w, m, n}\right)^{2}}{2 \sigma_{w, m, n}^{2}}\right\},
\end{aligned}
$$


where $\mu_{w, m, n}$ and $\sigma_{w, m, n}$ are the mean (location parameter) and standard deviation (scale parameter) of the logarithmic value of the daily travel distance, respectively.

At the mode value (i.e., the most frequent daily travel distance) of the log-normal distribution, $x_{w, m, n}^{*}$, the condition $\partial f\left(x_{w, m, n}^{*}\right) / \partial x_{w, m, n}=0$ is satisfied. Hence, the following equation can be obtained from this condition:

$$
\mu_{w, m, n}=\ln x_{w, m, n}^{*}+\sigma_{w, m, n}^{2} .
$$

Similar to the case of the Weibull distribution, the probability of traveling more than twice the most frequent daily travel distance can be expressed as follows:

$$
\frac{y_{w, m, n}^{\prime}}{y_{w, m, n}}=1-\Phi\left(\frac{\ln 2 x_{w, m, n}^{*}-\ln x_{w, m, n}^{*}-\sigma_{w, m, n}^{2}}{\sigma_{w, m, n}}\right)
$$

$$
=1-\Phi\left(\frac{\ln 2-\sigma_{w, m, n}^{2}}{\sigma_{w, m, n}}\right)
$$

where $\Phi(\cdot)$ is the cumulative distribution function of the standard normal distribution. Therefore, we can obtain the quadratic equation regarding the scale parameter $\sigma_{w, m, n}$ as follows:

$$
\frac{\ln 2-\sigma_{w, m, n}^{2}}{\sigma_{w, m, n}}=\Phi^{-1}\left(1-\frac{y_{w, m, n}^{\prime}}{y_{w, m, n}}\right)
$$

By solving the above equation, the value of $\sigma_{w, m, n}$ can be obtained analytically. Then, the location parameter $\mu_{w, m, n}$ can be obtained by using (7). Since the value of $\sigma_{w, m, n}$ must be positive, it can be obtained as follows:

$$
\sigma_{w, m, n}=\frac{-\Phi^{-1}\left(1-y_{w, m, n}^{\prime} / y_{w, m, n}\right)+\sqrt{\Phi^{-1}\left(1-y_{w, m, n}^{\prime} / y_{w, m, n}\right)^{2}+4 \ln 2}}{2} .
$$

Again, similar to the case of the Weibull distribution, it is assumed that an EV is fully charged at home in the morning and then is not recharged until the end of the day. In this case, the probability that an EV with range $R$ experiences battery depletion, $P_{\text {Depletion }}^{L N}\left(R \mid \mu_{w, m, n}, \sigma_{w, m, n}\right)$, is calculated as follows:

$$
\begin{aligned}
& P_{\text {Depletion }}^{L N}\left(R \mid \mu_{w, m, n}, \sigma_{w, m, n}\right) \\
& \quad=1-\Phi\left(\frac{\ln R-\mu_{w, m, n}}{\sigma_{w, m, n}}\right) .
\end{aligned}
$$

\subsubsection{Analysis of the Tolerable Probability of Battery Depletion.} The previous subsection described the methodology for calculating the battery depletion probability. The probability of the EV range desired by each household is the tolerable probability of battery depletion for the household. Figure 3 shows the distribution of the calculated tolerable probability of battery depletion. In the calculation, it is assumed that the car used most frequently in each household is replaced with an EV. In addition, the figure illustrates the distribution with and without the preferred deployment of charging stations. It clarifies that around $80 \%$ of respondents have a tolerable probability of less than $0.1 \%$. This finding means that many respondents expect a very low probability of battery depletion compared with their daily travel distance. Around 10\% of respondents indicate a relatively high tolerable probability of battery depletion, $10 \%$ to $50 \%$. The comparison between the two distribution functions, Weibull and log-normal, shows that the log-normal distribution has a lower tolerable probability of battery depletion because of its higher skewness. For both distribution functions, the distribution shifts in the direction of the higher probability when the preferred deployment of charging stations is implemented. The average tolerable probabilities of battery depletion are $2.18 \%$ for log-normal distribution and 2.61\% for Weibull distribution without the preferred deployment. These values shift to $2.82 \%$ and $4.91 \%$ with the preferred deployment, respectively. This result shows that the deployment of charging stations can reduce range anxiety and make people accept the short range of an EV.

4.2. Desired Range Model and Analysis. If changes in the desired EV range can be modeled, the effects of varying charging station deployment can be incorporated into the analysis of EV purchasing intentions. For the parameter estimation, the desired EV ranges obtained in the questionnaire can be used. Two answers are obtained from each household (i.e., the answers for with and without the preferred deployment of charging stations). To consider the dependency between these two answers from the same household, a bivariate probabilistic distribution should be applied.

4.2.1. Desired Range Model Based on a Bivariate Weibull Distribution. A bivariate Weibull distribution is applied in some studies of statistics or relatability engineering [40-44]. Based on Hougaard [41], for two random variables, $R_{1}$ and $R_{2}$, which correlate with each other and follow a Weibull distribution, the survival function (complementary cumulative distribution function) $\bar{F}_{\mathrm{BW}}\left(r_{1}, r_{2} \mid \lambda_{1}, \lambda_{2}, k_{1}, k_{2}, \gamma\right)$ can be expressed as follows:

$$
\begin{gathered}
\bar{F}_{\mathrm{BW}}\left(r_{1}, r_{2} \mid \lambda_{1}, \lambda_{2}, k_{1}, k_{2}, \gamma\right)=P\left(R_{1}>r_{1}, R_{2}>r_{2}\right) \\
=\exp \left[-\left\{\left(\frac{r_{1}}{\lambda_{1}}\right)^{k_{1} / \gamma}+\left(\frac{r_{2}}{\lambda_{2}}\right)^{k_{2} / \gamma}\right\}^{\gamma}\right],
\end{gathered}
$$

where $\lambda_{1}$ and $\lambda_{2}$ are scale parameters, $k_{1}$ and $k_{2}$ are shape parameters, and $\gamma$ is the joint parameter for considering the correlation. 


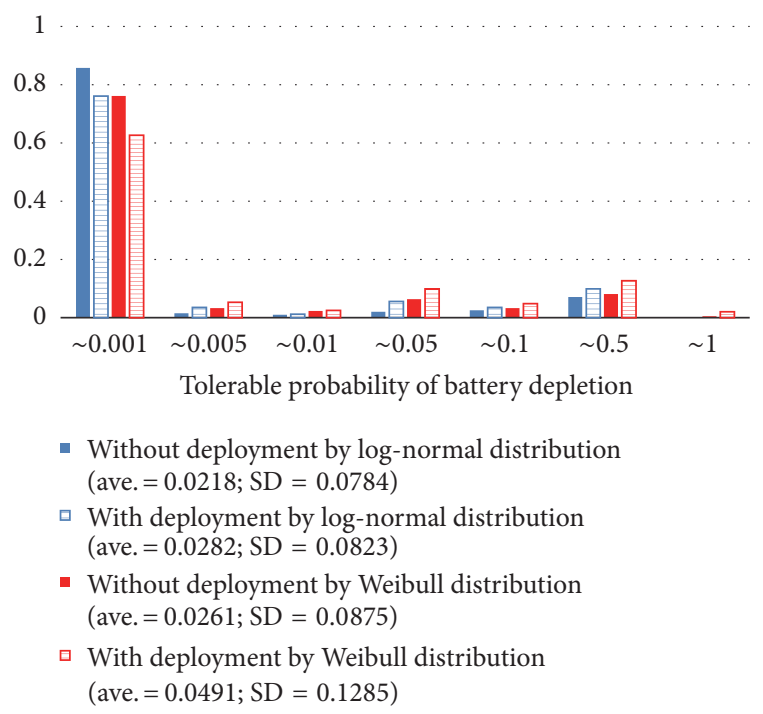

FIGURE 3: Distribution of the tolerable probability of battery depletion.

In this study, the answers about the desired EV range are chosen from nine range categories. Then, the probability that household $n$ desires range $r_{0, k-1} \sim r_{0, k}$ without deployment and $r_{1, j-1} \sim r_{1, j}$ with deployment, $P_{\mathrm{BW}}\left(r_{0, k-1}<R_{0, n}^{*} \leq r_{0, k}, r_{1, j-1}<\right.$ $\left.R_{1, n}^{*} \leq r_{1, j}\right)$, can be expressed as follows:

$$
\begin{aligned}
P_{\mathrm{BW}} & \left(r_{0, k-1}<R_{0, n}^{*} \leq r_{0, k}, r_{1, j-1}<R_{1, n}^{*} \leq r_{1, j}\right) \\
= & \bar{F}_{\mathrm{BW}}\left(r_{0, k-1}, r_{1, j-1} \mid \lambda_{1, n}, \lambda_{2, n}, k_{1, n}, k_{2, n}, \gamma\right) \\
& -\bar{F}_{\mathrm{BW}}\left(r_{0, k-1}, r_{1, j} \mid \lambda_{1, n}, \lambda_{2, n}, k_{1, n}, k_{2, n}, \gamma\right) \\
& -\bar{F}_{\mathrm{BW}}\left(r_{0, k}, r_{1, j-1} \mid \lambda_{1, n}, \lambda_{2, n}, k_{1, n}, k_{2, n}, \gamma\right) \\
& +\bar{F}_{\mathrm{BW}}\left(r_{0, k}, r_{1, j} \mid \lambda_{1, n}, \lambda_{2, n}, k_{1, n}, k_{2, n}, \gamma\right),
\end{aligned}
$$

where $R_{i, n}^{*}$ is the desired EV range for deployment condition $i$, which takes a value of zero for the situation without the preferred deployment and a value of one for that with the preferred deployment. To satisfy the positive value of the scale parameter $\lambda_{i, n}$ and shape parameter $k_{i, n}$, we formulate them as follows:

$$
\begin{aligned}
& \lambda_{i, n}=\exp \left(\boldsymbol{\beta}^{\mathrm{BW}} \mathbf{x}_{i, n}^{\mathrm{BW}}\right), \\
& k_{i, n}=\exp \left(\boldsymbol{\alpha}^{\mathrm{BW}} \mathbf{y}_{i, n}^{\mathrm{BW}}\right),
\end{aligned}
$$

where $\mathbf{x}_{i n}^{\mathrm{BW}}$ and $\mathbf{y}_{i, n}^{\mathrm{BW}}$ are the vectors of the explanatory variables for the scale parameter and shape parameter for deployment condition $i$, respectively. $\boldsymbol{\beta}^{\mathrm{BW}}$ and $\boldsymbol{\alpha}^{\mathrm{BW}}$ are the vectors of the unknown parameters for them. In this case, the expected value of the desired $\mathrm{EV}$ range is expressed as follows:

$$
E_{\mathrm{BW}}\left[R_{i, n}^{*}\right]=\lambda_{i, n} \Gamma\left(1+\frac{1}{k_{i, n}}\right)
$$

where $\Gamma(\cdot)$ is a gamma function. By incorporating the explanatory variables on the deployment condition of charging stations into (14a) and (14b), we can obtain the desired range of each household for that deployment condition through (15).

4.2.2. Desired Range Model Based on a Bivariate Log-Normal Distribution. In the case of the log-normal distribution, the probability that household $n$ desires range $r_{0, k-1} \sim r_{0, k}$ without the preferred deployment of charging stations and $r_{1, j-1} \sim r_{1, j}$ with the deployment, $P_{\mathrm{BLN}}\left(r_{0, k-1}<R_{0, n}^{*} \leq r_{0, k}, r_{1, j-1}<R_{1, n}^{*} \leq\right.$ $\left.r_{1, j}\right)$, can be expressed as follows [45, 46]:

$$
\begin{aligned}
P_{\mathrm{BLN}} & \left(r_{0, k-1}<R_{0, n}^{*} \leq r_{0, k}, r_{1, j-1}<R_{1, n}^{*} \leq r_{1, j}\right) \\
= & F_{\mathrm{BLN}}\left(\frac{\ln \left(r_{0, k}\right)-\mu_{0, n}}{\sigma_{0, n}}, \frac{\ln \left(r_{1, j}\right)-\mu_{1, n}}{\sigma_{1, n}}, \rho\right) \\
& -F_{\mathrm{BLN}}\left(\frac{\ln \left(r_{0, k-1}\right)-\mu_{0, n}}{\sigma_{0, n}}, \frac{\ln \left(r_{1, j}\right)-\mu_{1, n}}{\sigma_{1, n}}, \rho\right) \\
& -F_{\mathrm{BLN}}\left(\frac{\ln \left(r_{0, k}\right)-\mu_{0, n}}{\sigma_{0, n}}, \frac{\ln \left(r_{1, j-1}\right)-\mu_{1, n}}{\sigma_{1, n}}, \rho\right) \\
& +F_{\mathrm{BLN}}\left(\frac{\ln \left(r_{0, k-1}\right)-\mu_{0, n}}{\sigma_{0, n}}, \frac{\ln \left(r_{1, j-1}\right)-\mu_{1, n}}{\sigma_{1, n}}, \rho\right),
\end{aligned}
$$

where $F_{\mathrm{BLN}}(\cdot)$ is the cumulative distribution function of the bivariate log-normal distribution. For deployment condition $i, \mu_{i, n}$ is the mean value (location parameter) and $\sigma_{i, n}$ is the standard deviation (scale parameter) of the logarithmic range value and $\rho$ is the correlation coefficient.

In this study, similar to the case of the Weibull distribution, $\mu_{i, n}$ and $\sigma_{i, n}$ are formulated as follows:

$$
\begin{aligned}
\mu_{i, n} & =\boldsymbol{\beta}^{\mathrm{BLN}} \mathbf{x}_{i n}^{\mathrm{BLN}}, \\
\sigma_{i, n} & =\exp \left(\boldsymbol{\alpha}^{\mathrm{BLN}} \mathbf{y}_{i, n}^{\mathrm{BLN}}\right) .
\end{aligned}
$$

In this case, the expected value of the desired EV range is expressed as follows:

$$
E_{\mathrm{BLN}}\left[R_{i, n}^{*}\right]=\exp \left(\mu_{i, n}+\frac{\sigma_{i, n}^{2}}{2}\right) .
$$

4.2.3. Estimation Results of the Desired Range Models. Table 2 shows the estimation results. To compare the effect of deploying charging stations at different locations, the parameters of the deployment ratios are retained even if they are insignificant. The negative parameter estimates for $\mu_{i, n}$ and $\lambda_{i, n}$ decrease the desired range. The table shows that the deployment locations have different effects. Deployment at large-scale retail facilities, parking places at the workplace, and public facilities reduces the desired EV range. Deployment at small-scale retail facilities and expressway rest areas also has a significant reducing effect. That is, the deployment 


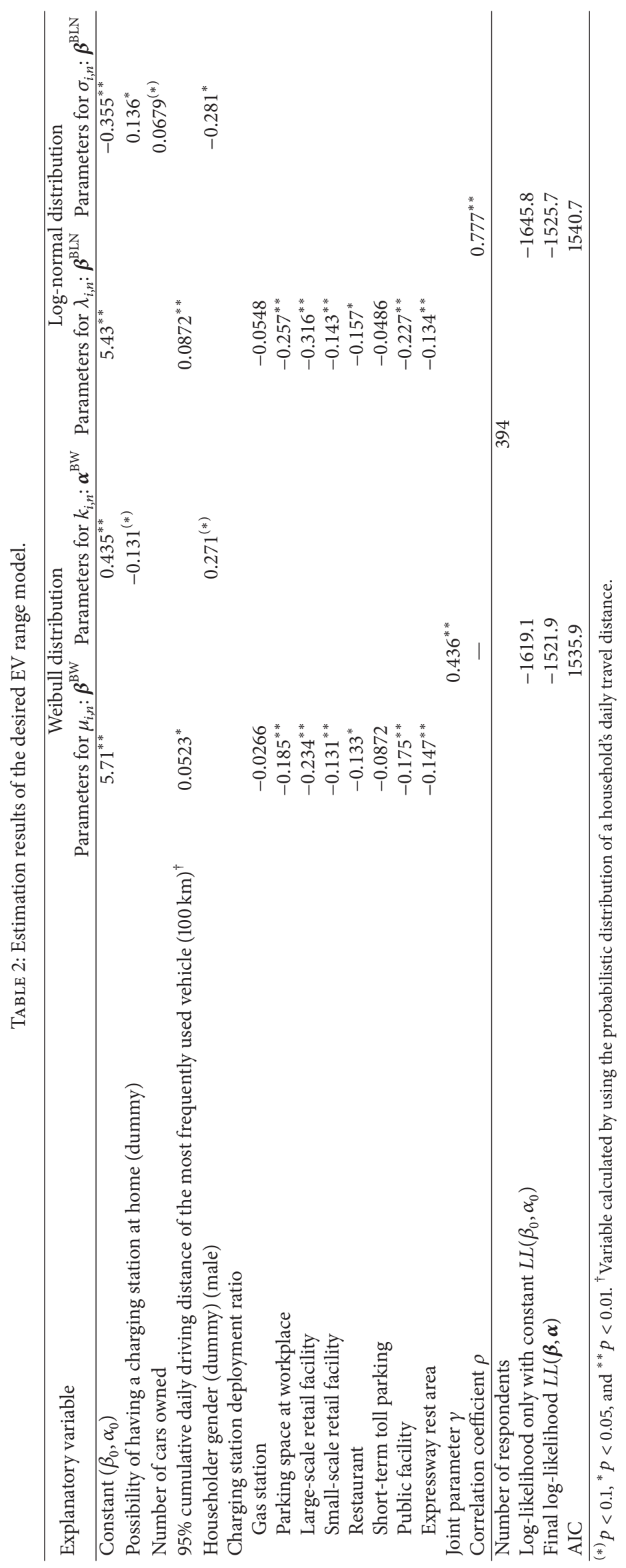


of charging stations at these locations can reduce range anxiety.

On the contrary, placing charging stations at gas stations, which are considered to be a typical deployment location, does not have a significant effect. A reasonable explanation for this is that charging takes longer than refueling, and hence gas stations may not be convenient to use in daily life. While the shape parameter $k_{i, n}$ of the Weibull distribution is the denominator in (15), the squared value of the scale parameter $\sigma_{i, n}$ of the log-normal distribution is the numerator in (18). That is, it is difficult to compare them. Nonetheless, it is found that a few variables have a significant impact. The possibility of having a charging station at home and the householder's gender tend to have an effect. Lastly, the AIC values show that the model based on the Weibull distribution has a slightly better fit.

\section{Analysis of EV Purchasing Intentions}

In this section, the EV purchasing intentions of households are analyzed. As mentioned above, two hypothetical EV features were shown and the preferences for purchasing an EV were asked for each. To take account of the dependency between the two answers from the same household, the probability of purchasing an EV is expressed by using a bivariate binary probit model as follows:

$$
\begin{aligned}
& U_{1, n}=V_{1, n}+\xi_{1, n}=\gamma \mathbf{z}_{1, n}+\xi_{1, n}, \\
& h_{1, n}= \begin{cases}1 & \text { if } U_{1, n}>0 \\
0 & \text { if } U_{1, n} \leq 0,\end{cases} \\
& U_{2, n}=V_{2, n}+\xi_{2, n}=\gamma^{\prime}+\gamma \mathbf{z}_{2, n}+\xi_{2, n}, \\
& h_{2, n}= \begin{cases}1 & \text { if } U_{2, n}>0 \\
0 & \text { if } U_{2, n} \leq 0,\end{cases}
\end{aligned}
$$

where, for household $n, U_{i, n}$ is the utility of purchasing the EV indicated in the $i$ th question (where $i$ is 1 or 2 in this study). $V_{i, n}$ and $\xi_{i, n}$ are its systematic and error terms, respectively. $\mathbf{z}_{i, n}$ and $\gamma$ are the vectors of the explanatory variables and parameters, respectively. $h_{i, n}$ is a dummy indicator taking the value of one when the decision is to purchase an EV and zero otherwise.

Although the same parameter estimates in $\gamma$ are expected for both utility functions, an additional parameter $\gamma^{\prime}$ is included to consider bias (e.g., due to fatigue) in the second question. In addition, the difference in error variances is taken into account. In this case, for example, the probability that household $n$ decides to purchase the EV for both SP questions can be expressed as follows:

$$
\begin{aligned}
P\left(h_{1, n}=1, h_{2, n}=1\right) & =P\left(U_{1, n}>0, U_{2, n}>0\right) \\
& =F_{\mathrm{BLN}}\left(V_{1, n}, V_{2, n} / r, \tau\right),
\end{aligned}
$$

where $r$ is the ratio of the standard deviations of the two error terms and $\tau$ is the correlation coefficient between them.
Table 3 shows the parameter estimates of the EV purchasing models. In the models, the EV range-related variables, namely, the desired range and probability of battery depletion, are calculated by using the Weibull distributionbased models established in Sections 4.1.1 and 4.1.2. This is because the Weibull distribution showed a better fit than the log-normal distribution in Section 4.1.2. In this analysis, to understand how households evaluate the EV range, three models with different range-related variables are considered: the logarithmic value of EV range (Model 1), the logarithmic ratio of the EV range to the desired EV range (Model 2), and the logarithmic value of 1 - the probability of battery depletion (Model 3). The probability of battery depletion for the EV range is calculated as a weighted average value based on the use frequency for all the cars owned in each household. The key explanatory variables such as price, charging time, and the deployment ratio of charging stations are retained even if their parameter estimates are not statistically significant. Lastly, the maximum saving in operational costs with the EV is calculated based on the daily driving distance distribution of the car used most frequently in the household.

From these results, we find that vehicle capacity and price are significant parameters for all models. These are therefore the key factors in deciding to purchase an EV. On the contrary, charging time, which is frequently focused on in the literature, is not significant for all models. A reasonable explanation for this may be that respondents may not take the disadvantages seriously into account when completing an SP questionnaire. Although the ratio of charging station deployment at expressway rest areas has a significant influence in some models, gas station deployment does not, as seen in the estimation result for the desired range (Table 2). That is, in the case of an EV purchasing decision, the deployment of charging stations at gas stations also has little attraction.

As for the estimates related to the EV range-related variables, they have significant parameters for all models, and it is shown that a longer range enhances EV purchasing intention. Model 3 shows that the possibility of charging at home dissipates the anxiety of battery depletion. From the point of view of the model fitness indices, however, Model 3 is inferior to Model 1 and Model 2. Model 1 shows a slightly better fit than Model 2. Thus, these results demonstrate that households do not consider the probability of battery depletion when deciding to purchase an EV. Instead, they tend to evaluate the EV's range by itself or directly compare it with their desired range.

\section{Conclusion and Future Research}

In this research, the authors investigated concerns about an EV's range and battery depletion by using the data obtained from a questionnaire survey. Specifically, a probabilistic distribution of the daily travel distance and a model of the desired EV range were developed and analyzed. In addition, $\mathrm{EV}$ purchasing decision models were developed and analyzed.

The results of this analysis demonstrate that a risk of battery depletion of about $2 \%$ is tolerated without the preferred deployment of charging stations, and this rises to 
TABLE 3: Estimation results of the three EV purchase models.

\begin{tabular}{|c|c|c|c|}
\hline & Model 1 & Model 2 & Model 3 \\
\hline Constant & -0.354 & $-1.07^{*}$ & -0.411 \\
\hline Capacity & $0.122^{* *}$ & $0.119^{* *}$ & $0.132^{* *}$ \\
\hline $\ln$ (price) $(1000$ yen $)$ & $-1.12^{* *}$ & $-1.09^{* *}$ & $-1.15^{* *}$ \\
\hline $\ln$ (time for charging) (hours) & -0.180 & -0.151 & -0.244 \\
\hline \multicolumn{4}{|l|}{ Vehicle range-related variables } \\
\hline $\ln ($ vehicle range $)(100 \mathrm{~km})$ & $0.443^{* *}$ & & \\
\hline $\ln ($ vehicle range/desired vehicle range $)(100 \mathrm{~km})^{\dagger}$ & & $0.312^{* *}$ & \\
\hline $\ln (1-\text { probability of battery depletion })^{\dagger}$ & & & $1.33^{*}$ \\
\hline Interaction with the possibility of charging at home (dummy) & & & $-1.35^{*}$ \\
\hline \multicolumn{4}{|l|}{ Charging station deployment rate } \\
\hline Gas stations & 0.0260 & 0.0596 & 0.139 \\
\hline Expressway rest areas & $0.264^{(*)}$ & 0.237 & $0.393^{*}$ \\
\hline Maximum saved operation cost with an EV (yen/month) ${ }^{\dagger}$ & $0.115^{(*)}$ & $0.107^{(*)}$ & $0.179^{*}$ \\
\hline Constant (second answer) & -0.224 & -0.178 & -0.280 \\
\hline Standard deviation of the error term (second answer) & $1.20^{* *}$ & $1.15^{* *}$ & $1.45^{* *}$ \\
\hline Correlation coefficient & $0.411^{* *}$ & $0.390^{* *}$ & $0.352^{* *}$ \\
\hline Number of samples & & 394 & \\
\hline Log-likelihood only with constant $L L(0)$ & & -546.2 & \\
\hline Final log-likelihood $L L(\boldsymbol{\beta}, \boldsymbol{\alpha})$ & -467.9 & -466.2 & -474.7 \\
\hline Rho-squared value & 0.143 & 0.146 & 0.131 \\
\hline Adjusted rho-squared value & 0.123 & 0.126 & 0.109 \\
\hline
\end{tabular}

around $3-5 \%$ with deployment at the preferred locations. The Weibull and log-normal distributions were applied to the desired EV range model. The estimation results show that the applicability of the Weibull distribution is slightly higher. In addition, it is found that the deployment of charging stations in locations where drivers tend to remain parked for longer periods such as large-scale retail facilities and workplaces relieves the range anxiety of EVs and thus reduces the desired range. Moreover, making charging available at gas stations has no significant effect of reducing the desired EV range. Moreover, based on the estimation result of the EV purchasing decision model, charging time does not have a significant effect on the decision to purchase an EV. Lastly, it is found that households do not consider the probability of battery depletion when deciding to purchase an EV; rather, they tend to evaluate the EV's range by itself or compare it with their desired range.

Future research on this topic should consider a more detailed investigation of EV purchasing behavior in two main directions: the purchase of an additional vehicle and the purchase of a replacement vehicle. When replacing an existing vehicle, the household must decide which existing car to release. Further, it can be expected that the usage of cars in a household will change after purchasing a new EV. The authors will continue to analyze the data with respect to these points.

\section{Conflicts of Interest}

The authors declare that there are no conflicts of interest regarding the publication of this paper.

\section{Acknowledgments}

This research was financially supported by the Japan Society for the Promotion of Science through the Grant-in-Aid for Scientific Research (no. 16H02367) and the authors gratefully acknowledge this support.

\section{References}

[1] Next Generation Vehicle Promotion Center website, https:// www.cev-pc.or.jp/english/, 2016.

[2] S. Shepherd, P. Bonsall, and G. Harrison, "Factors affecting future demand for electric vehicles: A model based study," Transport Policy, vol. 20, pp. 62-74, 2012.

[3] T. Gnann, P. Plötz, A. Kühn, and M. Wietschel, "Modelling market diffusion of electric vehicles with real world driving data - German market and policy options," Transportation Research Part A: Policy and Practice, vol. 77, pp. 95-112, 2015.

[4] S. Bubeck, J. Tomaschek, and U. Fahl, "Perspectives of electric mobility: Total cost of ownership of electric vehicles in Germany," Transport Policy, vol. 50, pp. 63-77, 2016. 
[5] M. Mohamed, C. Higgins, M. Ferguson, and P. Kanaroglou, "Identifying and characterizing potential electric vehicle adopters in Canada: A two-stage modelling approach," Transport Policy, vol. 52, pp. 100-112, 2016.

[6] C. Rudolph, "How may incentives for electric cars affect purchase decisions?" Transport Policy, vol. 52, pp. 113-120, 2016.

[7] S. Wang, J. Fan, D. Zhao, S. Yang, and Y. Fu, "Predicting consumers' intention to adopt hybrid electric vehicles: using an extended version of the theory of planned behavior model," Transportation, vol. 43, no. 1, pp. 123-143, 2016.

[8] S. Beggs, S. Cardell, and J. Hausman, "Assessing the potential demand for electric cars," Journal of Econometrics, vol. 16, no. 1, pp. 1-19, 1981.

[9] T. Franke and J. F. Krems, "What drives range preferences in electric vehicle users?” Transport Policy, vol. 30, pp. 56-62, 2013.

[10] H. A. Bonges and A. C. Lusk, "Addressing electric vehicle (EV) sales and range anxiety through parking layout, policy and regulation," Transportation Research Part A: Policy and Practice, vol. 83, pp. 63-73, 2016.

[11] T. Franke, I. Neumann, F. Bühler, P. Cocron, and J. F. Krems, "Experiencing range in an electric vehicle: understanding psychological barriers," Applied Psychology, vol. 61, no. 3, pp. 368391, 2012.

[12] A. Adepetu and S. Keshav, "The relative importance of price and driving range on electric vehicle adoption: Los Angeles case study," Transportation, vol. 44, no. 2, pp. 353-373, 2017.

[13] Nissan Motor Co., https://www3.nissan.co.jp/en.html, 2017.

[14] S. Greaves, H. Backman, and A. B. Ellison, "An empirical assessment of the feasibility of battery electric vehicles for dayto-day driving," Transportation Research Part A: Policy and Practice, vol. 66, no. 1, pp. 226-237, 2014.

[15] D. L. Greene, "Estimating daily vehicle usage distributions and the implications for limited-range vehicles," Transportation Research Part B: Methodological, vol. 19, no. 4, pp. 347-358, 1985.

[16] N. S. Pearre, W. Kempton, R. L. Guensler, and V. V. Elango, "Electric vehicles: How much range is required for a day's driving?" Transportation Research Part C: Emerging Technologies, vol. 19, no. 6, pp. 1171-1184, 2011.

[17] J. Woodjack, D. Garas, A. Lentz, T. Turrentine, G. Tal, and M. Nicholas, "Consumer perceptions and use of driving distance of electric vehicles," Transportation Research Record, no. 2287, pp. 1-8, 2012.

[18] N. Rauh, T. Franke, and J. F. Krems, "Understanding the impact of electric vehicle driving experience on range anxiety," Human Factors: The Journal of the Human Factors and Ergonomics Society, vol. 57, no. 1, pp. 177-187, 2015.

[19] G. Tal, M. A. Nicholas, J. Davies, and J. Woodjack, "Charging behavior impacts on electric vehicle miles traveled: who is not plugging in?" Transportation Research Record, vol. 2454, pp. 5360, 2014.

[20] J. Stark, C. Link, D. Simic, and T. Bäuml, "Required range of electric vehicles - An analysis of longitudinal mobility data," IET Intelligent Transport Systems, vol. 9, no. 2, pp. 119-127, 2015.

[21] X.-H. Sun, T. Yamamoto, and T. Morikawa, "Stochastic frontier analysis of excess access to mid-trip battery electric vehicle fast charging," Transportation Research Part D: Transport and Environment, vol. 34, pp. 83-94, 2015.

[22] Z. Li, S. Jiang, J. Dong, S. Wang, Z. Ming, and L. Li, "Battery capacity design for electric vehicles considering the diversity of daily vehicles miles traveled," Transportation Research Part C: Emerging Technologies, vol. 72, pp. 272-282, 2016.
[23] D. S. Bunch, M. Bradley, T. F. Golob, R. Kitamura, and G. P. Occhiuzzo, "Demand for clean-fuel vehicles in California: A discrete-choice stated preference pilot project," Transportation Research Part A: Policy and Practice, vol. 27, no. 3, pp. 237-253, 1993.

[24] K. S. Kurani, T. Turrentine, and D. Sperling, "Demand for electric vehicles in hybrid households: an exploratory analysis," Transport Policy, vol. 1, no. 4, pp. 244-256, 1994.

[25] E. Chéron and M. Zins, "Electric vehicle purchasing intentions: The concern over battery charge duration," Transportation Research Part A: Policy and Practice, vol. 31, no. 3, pp. 235-243, 1997.

[26] T. F. Golob and J. Gould, "Projecting use of electric vehicles from household vehicle trials," Transportation Research Part B: Methodological, vol. 32, no. 7, pp. 441-454, 1998.

[27] P. Plötz, U. Schneider, J. Globisch, and E. Dütschke, "Who will buy electric vehicles? Identifying early adopters in Germany," Transportation Research Part A: Policy and Practice, vol. 67, pp. 96-109, 2014.

[28] A. F. Jensen, E. Cherchi, and J. de Dios Ortúzar, "A long panel survey to elicit variation in preferences and attitudes in the choice of electric vehicles," Transportation, vol. 41, no. 5, pp. 973-993, 2014.

[29] S. M. Skippon, N. Kinnear, L. Lloyd, and J. Stannard, "How experience of use influences mass-market drivers' willingness to consider a battery electric vehicle: a randomised controlled trial," Transportation Research Part A: Policy and Practice, vol. 92, pp. 26-42, 2016.

[30] D. Kim, J. Ko, and Y. Park, "Factors affecting electric vehicle sharing program participants' attitudes about car ownership and program participation," Transportation Research Part D: Transport and Environment, vol. 36, pp. 96-106, 2015.

[31] B. Junquera, B. Moreno, and R. Álvarez, "Analyzing consumer attitudes towards electric vehicle purchasing intentions in Spain: Technological limitations and vehicle confidence," Technological Forecasting \& Social Change, vol. 109, pp. 6-14, 2016.

[32] M. J. Beck, J. M. Rose, and S. P. Greaves, "I can't believe your attitude: a joint estimation of best worst attitudes and electric vehicle choice," Transportation, vol. 44, no. 4, pp. 753-772, 2016.

[33] J. Axsen, S. Goldberg, and J. Bailey, "How might potential future plug-in electric vehicle buyers differ from current "Pioneer" owners?" Transportation Research Part D: Transport and Environment, vol. 47, pp. 357-370, 2016.

[34] T. Franke, M. Günther, M. Trantow, and J. F. Krems, "Does this range suit me? Range satisfaction of battery electric vehicle users," Applied Ergonomics, vol. 65, pp. 191-199, 2017.

[35] R. J. Javid and A. Nejat, "A comprehensive model of regional electric vehicle adoption and penetration," Transport Policy, vol. 54, pp. 30-42, 2017.

[36] J. Yang, T. Miwa, T. Morikawa, and T. Yamamoto, "Examining the preference of electric vehicles purchasing behavior using stated preference data," in Proceedings of the Journal of International City Planning, pp. 213-223, 2012.

[37] J. Yang, T. Miwa, T. Morikawa, and T. Yamamoto, "A discretecontinuous model for analyzing the ownership and usage of electric vehicles using stated preference data," Journal of the Eastern Asia Society for Transportation Studies, vol. 10, pp. 499514, 2013.

[38] Z. Lin, J. Dong, C. Liu, and D. Greene, "Estimation of energy use by plug-in hybrid electric vehicles," Transportation Research Record, no. 2287, pp. 37-43, 2012. 
[39] H. Wang, L. Wu, C. Hou, and M. Ouyang, "A GPS-based research on driving range and patterns of private passenger vehicle in Beijing," in Proceedings of EVS27, 2013.

[40] A. W. Marshall and I. Olkin, "A multivariate exponential distribution," Journal of the American Statistical Association, vol. 62, pp. 30-44, 1967.

[41] P. Hougaard, "A class of multivariate failure time distributions," Biometrika, vol. 73, no. 3, pp. 671-678, 1986.

[42] D. Kundu and A. K. Dey, "Estimating the parameters of the Marshall-Olkin bivariate Weibull distribution by EM algorithm," Computational Statistics \& Data Analysis, vol. 53, no. 4, pp. 956-965, 2009.

[43] D. D. Hanagal, "Weibull extension of bivariate exponential regression model with different frailty distributions," Statistical Papers, vol. 50, no. 1, pp. 29-49, 2009.

[44] K. K. Jose, M. M. Ristic, and A. Joseph, "Marshall-Olkin bivariate Weibull distributions and processes," Statistical Papers, vol. 52, no. 4, pp. 789-798, 2011.

[45] C. Bhat, "Estimation of travel demand models with grouped and missing income data," Transportation Research Record, vol. 1443, pp. 45-53, 1994.

[46] W. H. Greene and D. A. Hensher, Modeling ordered choices, Cambridge University Press, Cambridge, 2010. 


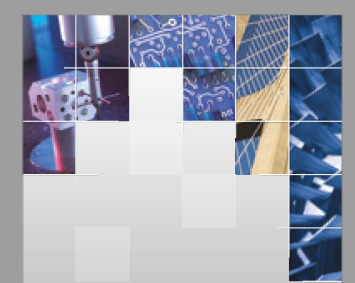

\section{Enfincering}
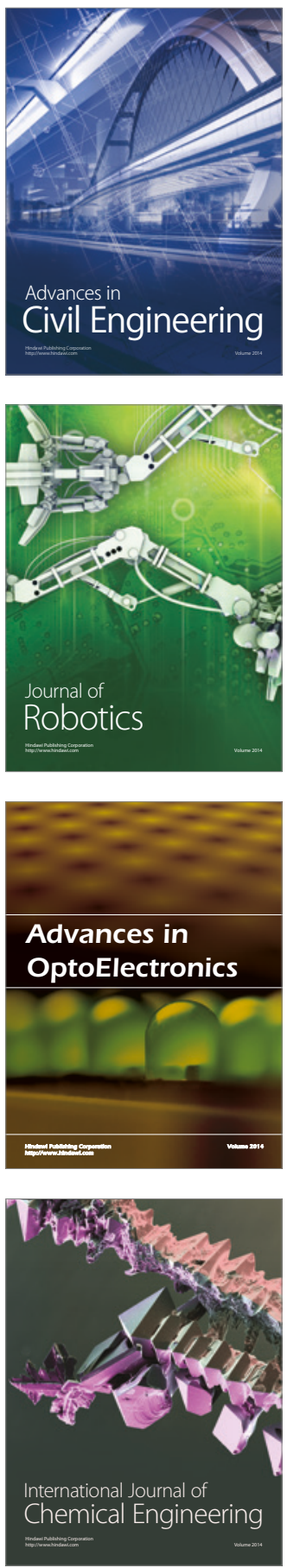

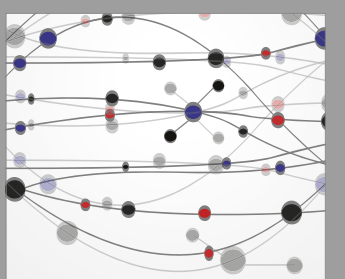

The Scientific World Journal

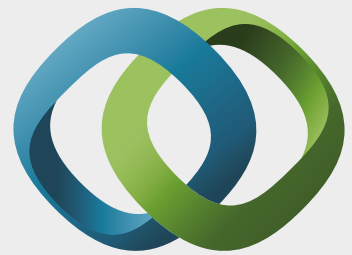

\section{Hindawi}

Submit your manuscripts at

https://www.hindawi.com
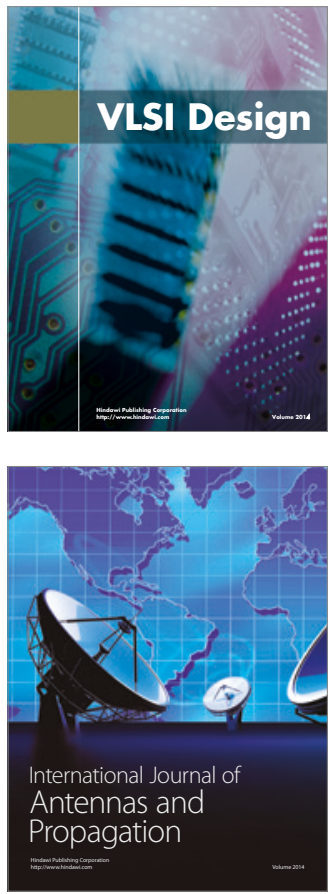

\section{Rotating}

Machinery
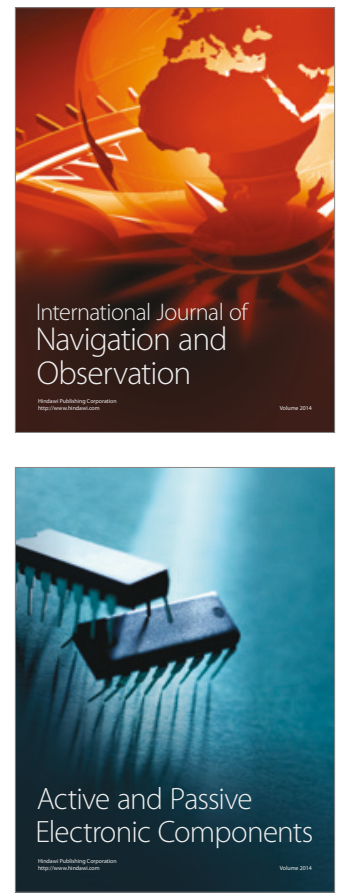
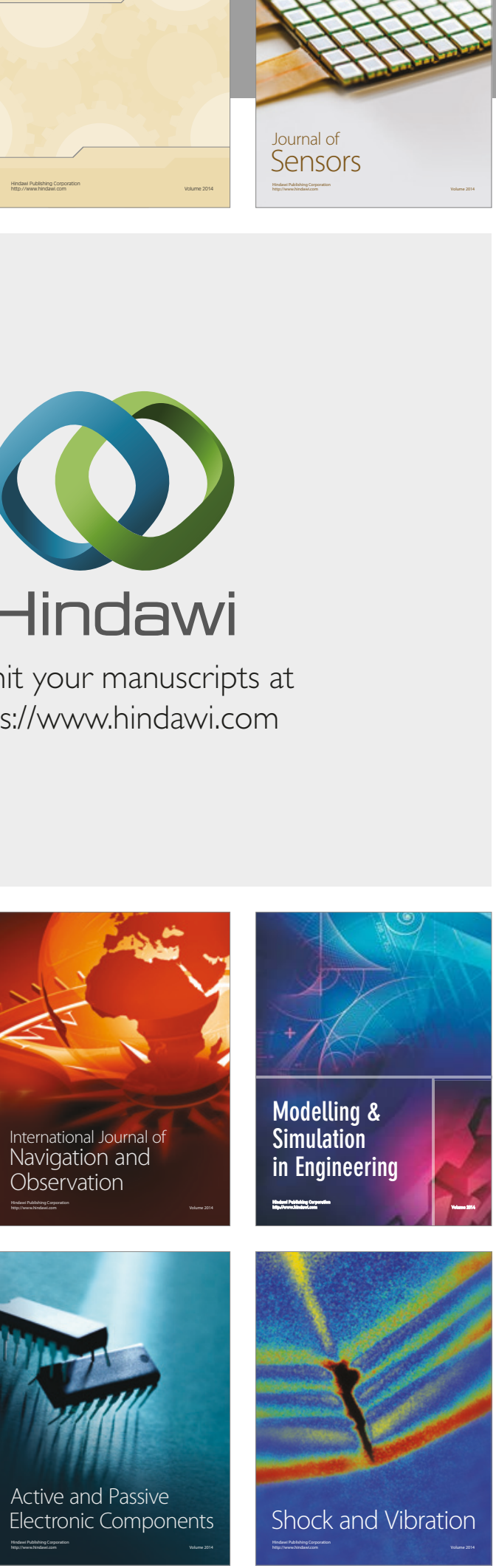
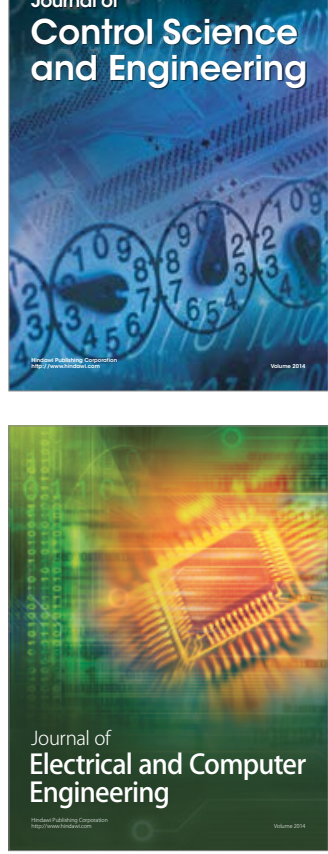

Distributed

Journal of

Control Science

and Engineering
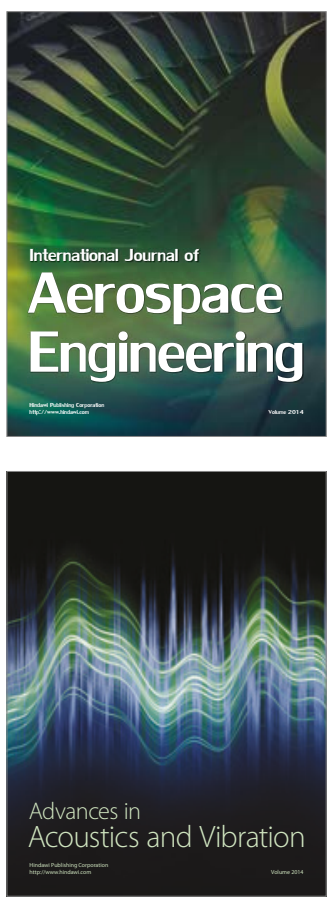

Sensor Networks 